2 Hussain B, Saleh GM, Sivaprasad S, Hammond CJ. Changing from Snellen to LogMAR: debate or delay? Clin Exp Ophthalmol 2006; 34: 6-8.

3 Bailey I, Lovie J. New design principles for visual acuity letter charts. Am J Optom Physiol Opt 1976; 53: 740-745.

4 Laidlaw DAH, Abbott A, Rosser DA. Development of a clinically feasible logMAR alternative to the Snellen chart: performance of the compact reduced logMAR visual acuity chart in amblyopic children. Br J Ophthalmol 2003; 87: 1232-1234.

5 Talks SJ, Setty R, Clarke L. The incidence and outcome of photodynamic therapy for macular degeneration in the Northern region of the UK. Eye 2004; 18: 588-594.

6 Bressler NM. Photodynamic therapy of subfoveal choroidal neovascularization in age-related macular degeneration with verteporfin: two-year results of 2 randomized clinical trials-tap report 2. Arch Ophthalmol 2001; 119: 198-207.

E Doyle and AG Casswell

Sussex Eye Hospital, Eastern Road, Brighton BN2 5BF, UK

Correspondence: E Doyle,

Tel: + 44 (0) 7799448543;

Fax: + 44 (0) 8714338914

E-mail: EdRachie@btinternet.com

The authors have no commercial interests in any products mentioned in this article

Eye (2007) 21, 284-285. doi:10.1038/sj.eye.6702532;

published online 4 August 2006

Sir,

\section{Reply to Doyle et al}

We would like to thank Doyle et al for their interest in our reported series of four cases. ${ }^{1}$ We agree that logMAR visual acuity measurements should be encouraged especially in situations where measurements using a Snellen chart may not be sensitive enough to recognise a change in the acuity. In keeping with the standard practice we routinely record $\log$ MAR visual acuities for all patients undergoing PDT. In the text of our report we have reported the decline in visual acuity in terms of the drop in the number of letters read on the $\log$ MAR chart. This is similar to the way results were summarised in the TAP study ${ }^{2}$ where reduction of visual acuity by fewer than 15 letters over a 24-month period was considered as beneficial effect of the treatment. Furthermore, although one of our patients lost only 14 letters, this happened rapidly over a 9-month period in spite of closure of the CNV which is not strictly comparable with the beneficial outcome reported in the TAP study.

Doyle et al have said that nowhere in the paper is the actual $\log$ MAR visual acuity shown. We would like to draw their attention to Figures 2 and 3 in our report that clearly mention the actual $\log$ MAR visual acuity values for that case. We feel that using the reciprocal of actual $\log$ MAR values appropriately illustrates graphically in Figure 1 the steep decline in visual acuity as a downward slope. We do not think that adding a table showing logMAR visual acuities would have added to our message from this small case series that in certain cases visual acuity could decline fairly rapidly in spite of a 'successful' PDT treatment.

\section{References}

1 Bhatnagar A, Musadiq M, Yang YC. Late-onset visual decline following successful treatment of subfoveal choroidal neovascularisation with photodynamic therapy. Eye 2006; 20: 491-493.

2 Treatment of Age-Related Macular Degeneration with Photodynamic Therapy (TAP) Study Group. Photodynamic therapy of subfoveal choroidal neovascularisation in age related macular degeneration with verteporfin. Two-year results of two randomised clinical trials-TAP report 2. Arch Ophthalmol 2001; 119: 198-207.

\section{A Bhatnagar, M Musadiq and YC Yang}

Wolverhampton Eye Infirmary, Compton Road, Wolverhampton, WV3 9QR, UK

Correspondence: A Bhatnagar, Wolverhampton Eye Infirmary, Compton Road, Wolverhampton, WV3 9QR UK

Tel: + 447958 582871;

Fax: + 441902645018.

E-mail: bhatnagar_ajay@btopenworld.com

Eye (2007) 21, 285. doi:10.1038/sj.eye.6702534; published online 4 August 2006

Sir,

Intravitreal triamcinolone staining observation of residual undetached cortical vitreous after posterior vitreous detachment

The use of intravitreal triamcinolone to demonstrate areas of undetached vitreous ${ }^{1}$ has gained in popularity in recent years. The authors have not clarified whether their 
technique is also applicable for the identification (and removal) of residual or 'undetached' posterior hyaloid membrane.

The histology, immunohistochemistry, and ultrastructure of the posterior hyaloid membrane has been extensively described, ${ }^{2-6}$ and recent research has highlighted its role in the pathogenesis of many vitreoretinal disorders, including macular holes, cellophane maculopathy, macular pucker, and vitreomacular traction syndrome. ${ }^{4,5}$ It has also become clear that the presence or absence of a Weiss ring is insufficient for the diagnosis of complete separation of PHM. ${ }^{4,5}$

The authors allude to the possibility of 'undetached hyaloid fragments' being related to future epiretinal membrane (ERM) formation, but recent research suggests that the evidence for PHM in this role is particularly strong. ${ }^{5}$ Incomplete separation of the PHM, rather than the cortical gel it envelops, would explain why seven patients in the study developed ERM without any associated residual vitreous staining (with one developing severe macular pucker).

We support the authors' proposition that evaluation of the vitreo-retinal relationship in eyes with retinal detachment, 'even when there is apparent PVD', is of great clinical importance. However, although triamcinolone acetonide appears helpful in the demonstration of residual cortical vitreous, it is important to recognise that residual vitreous is merely a pointer to an underlying incomplete separation of the posterior hyaloid membrane.

\section{References}

1 Chen TY, Yang CM, Liu KR. Intravitreal triamcinolone staining observation of residual undetached cortical vitreous after posterior vitreous detachment. Eye 2006; 20: 423-427.

2 Zimmerman LE, Straatsma BR. Anatomic relationships of the retina to the vitreous body and to the pigment epithelium. In: Schepens CL (ed.) Importance of the Vitreous Body in Retina Surgery with Special Emphasis on Reoperations. CV Mosby Co: St Louis, 1960, pp 15-28.

3 Snead MP, Snead DR, Richards AJ, Harrison JB, Poulson AV Morris et al. Clinical, histological and ultrastructural studies of the posterior hyaloid membrane. Eye 2002; 16: 447-453.

4 Ang A, Snead DRJ, James S, Yeo YC, Satchi K, Morris AHC et al. A rationale for membrane peeling in the repair of stage 4 macular holes. Eye 2006; 20: 208-214.

5 Snead DR, Cullen N, James S, Poulson AV, Morris AH, Lukaris A et al. Hyperconvolution of the inner limiting membrane in vitreomaculopathies. Graefes Arch Clin Exp Ophthalmol 2004; 242(10): 853-862 (E-pub 7 October 2004).

6 Ang A, Poulson AV, Snead DR, Snead M. Posterior vitreous detachment: current concepts and management. Compr Ophthalmol Update 2005; 6(4): 167-175.
SJ Robbie and MP Snead

Addenbrooke's Hospital, Hills Road,

Cambridge CB2 2QQ, UK

Correspondence: SJ Robbie,

Tel: + 4407932647848 ;

Fax: + 4402077930071 .

E-mail: scott.robbie@addenbrookes.nhs.uk

Eye (2007) 21, 285-286. doi:10.1038/sj.eye.6702533;

published online 4 August 2006

Sir,

Reply to Robbie and Snead

We appreciate Robbie and Snead's comment on our article: 'Intravitreal triamcinolone staining observation of residual undetached cortical vitreous after posterior vitreous detachment'. In their comment, the authors made a distinction between posterior hyaloid membrane (PHM) and cortical gel it envelops, and questioned about whether our technique is also applicable for the identification (and removal) of residual or undetached PHM. Triamcinolone acetonide (TA)-assisted identification of undetached PHM utilizes the property of TA particles to adhere to the cortical vitreous for better visualization of the posterior vitreous membrane. ${ }^{1}$ Horio et $a l^{2}$ in their study of TA-assisted internal limiting membrane (ILM) peeling found, under light microscopy, TA particles adhered to the thin layer of the residual vitreous on the ILM. This finding suggests that only a thin layer of vitreous is required for TA to stain. We assume that most, if not all, residual vitreous fragments on the retina surface after recent onset PVD should contain at least a thin layer of vitreous and thus can be stained with TA. It is not known how frequent and on what conditions would 'naked' PHM exists.

Based on their histopathological studies of PHM and epiretinal membrane (ERM), 3,4 the authors suggest that the ERMs developed in seven cases in our series come from undetached PHM. Ultrastructural studies of ERMs found various types of cells within the membranes, including fibrous astrocytes, fibrocytes, macrophages, and even retinal pigment epithelial cells. The combination of cells in each membrane depends on clinical entities. ${ }^{5}$ In our study, 10 of 23 cases had macular TA staining, and none developed visible macular ERM, and those seven postoperative ERM cases did not had intraoperative TA staining in the corresponding area. Although PHM may be important in the formation of ERMs in other vitreoretinal diseases, such as stage 4 